Recibido: 16-06-2015

Aceptado: 08-07-2015

Palabras clave: comprensión lectora, inferencias, inferencia global, inferencia local, texto digital, texto impreso. Keywords: reading comptehension, inference, global inference, local inference, digital text, printed text.

Patricia Matilde Herrera Salazar I.E. Solidaridad III pattyherrera2008@gmail.com

\section{Niveles de inferencia de comprensión lectora según tipo de texto estímulo en estudiantes de educación secundaria *}

\section{Inferential level in reading comprehension depending different types of document on secondary students}

\section{Patricia Matilde Herrera Salazar}

RESUMEN. Se realizó un estudio con el fin de verificar la diferencia significativa en la comprensión lectora en el nivel inferencial entre los formatos impresos y digital. La investigación es una comparación de grupos con solo postest que se llevó a cabo en una institución educativa estatal de nivel secundario de Lima Metropolitana. Participaron 38 estudiantes seleccionados de 80 estudiantes del cuarto grado de secundaria, según los resultados del Test de Matrices Progresivas de Raven. Se aplicó un cuestionatio sobre hábito lector, un cuestionatio sobre su relación con los medios digitales y una prueba de comprensión lectora para medir el nivel inferencial a nivel local y global, en formato impreso y digital Los resultados permiten concluir que no se han encontrado diferencias significativas entre los datos obtenidos en ambos tipos de formato de lectura. Asimismo, se confirmó que la competencia digital no afecta de manera significativa el nivel de comprensión inferencial de los estudiantes.

ABSTRACT. A study was conducted with the purpose of verifying the significant difference in reading comprehension at an infetential level between print and digital formats. This research is a group comparison using only a posttest, conducted in a state-run secondary school in Lima. From the 80 students in year four, 38 were selected following the results of Raven's Progressive Matrices Test. A questionnaire on reading habits was applied, as well as a questionnaire on the students' relationship with digital media and a teading comprehension test measuring the inferential level locally and gobally, in both print and digital formats. The results lead to the conclusion that no significant differences were found between the data obtained in both reading formats. In addition, it was confirmed that digital competence does not significantly affect the level of students' inferential comprehension.

* Tesis para optar el Grado de Magister en Educación, UNIFE, 2015 
Alétheia 2015,3 (1) 61

\section{Introducción}

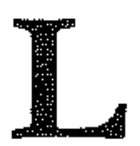

a globalización económica y el uso de las tecnologías de la información y de la comunicación (TIC), entre otros factores, ha traído consigo una transformación comunicativa, la cual también está modificando los sistemas de enseñanza aprendizaje. Esto ha promovido la incorpotación de nuevos recursos didácticos en entornos virtuales y el dictado de clases de manera virtual. De esta manera, una sesión de clase ya no solo depende de un pizarrón y de libros impresos. Ahora son los libros digitales.

Bajo estas circunstancias, un estudiante promedio cuya edad fluctúa entre los 14 y 17 años de edad ha nacido después de la implantación de la red y ha crecido rodeado de pantallas, teléfonos móviles y accesos a Google y Youtube, a diferenica con los inmigtantes digitales que han nacido antes y se educaron con papeles, lápices y libros. Por lo tanto, los adolescentes pertenecen a una sociedad donde predomina el uso de dispositivos electrónicos relacionados con el entorno virtual. Incluso, en el Internet pueden leer nuevos génetos electrónicos, los cuales se clasifican en sincrónicos como el chat, messenger, webcam o juegos de rol, y los asincrónicos como las páginas web, los blogs, los wikis, los correos electrónicos y las redes sociales. Es decir, los estudiantes de esta generación leen cada vez más textos digitalizados que impresos. Este panorama de la era virtual ha motivado que a partir de 2009, las pruebas PISA de ese año empezaran a evaluar la competencia en lectura electrónica.

La lectura en línea o virtual es la fuente inicial para muchos niños y jóvenes porque ellos prefieren acudir a fuentes como Wikipedia o Google para buscar temas de interés. De allí que enseñar a leer exclusivamente libros o documentos escritos en papel es considerado no adecuado o desfasado. Los estudiantes manejan bien las cuestiones mecánicas de las TIC, pero ignoran la arquitectura de la red, los criterios de selección de fuentes de investigación, etc. Finalmente, sostiene que leer en línea es más difícil que leer en papel porque carecen de actividades estratégicas para leer críticamente en línea.
La presente investigación de naturaleza pedagógica trata de encontrar las diferencias existentes entre la comprensión de textos impresos y la comprensión de textos digitalizados en el nivel inferencial en estudiantes de $4^{\circ}$ grado de secundaria de un colegio estatal mixto.

De acuerdo con Maqueo (2004, pp. 20-21) el modelo cognitivo es el que tiene mayor proyección hacia el futuro debido a que su interés es el estudio de las representaciones mentales (la atención, el recuerdo, la memoria, la percepción, la inteligencia, la concentración, el pensamiento, las ideas, conceptos y planes, la lengua, etc.), las cuales intervienen en modelos de estudio de la comprensión lectora.

Para Pinzás (1995, p. 15) la lectura es un proceso constructivo, interactivo, estratégico y metacognitivo porque a través de él, el lector va armando mentalmente un modelo de texto al cual se asigna una interpretación personal. Es así que, el lector, aparte de decodificar, detectar estructuras sintácticas y extraer significados de cada oración, debe identificar relaciones entre las diferentes partes del texto e interrelacionar con el texto según sus saberes previos. Conforme avanza la lectura, inferirá más relaciones implícitas. Si todo este proceso lo realiza con éxito, la representación mental creada a partir del texto leído permitirá recordarlo mejot y responder a las preguntas sobre el mismo. De esta manera, podrá decirse que ha sido comprendido.

La inferencia es la base de la actividad de la comprensión, ya que permiten integrar, a medida que la lectura avanza, los factores intratextuales y contextuales que interviene en el armado de la coherencia La inferencia local es la deducción realizada a partir de las relaciones de significados en el interior de un párrafo (mictoestructura del texto). Esta se refiere a las ideas principales, situaciones de causa-efecto, etc. La inferencia global se tealiza a nivel de todo el texto (macroestructura global) y alude al propósito o finalidad del texto, intención del autor, conclusión general, etc.

Para un mejor entendimiento del tema, Graesser y Kreuz (1993), citados por Escoriza (2003, p. 5) sostienen que las inferencias son producidas 


\section{UNIFÉ-EPG}

durante la comprensión del discurso escrito cuando las estructuras cognitivas son activadas y el contenido de estas es integrado en el significado construido en interacción con el discurso escrito. El proceso de comprensión lectora es, por lo tanto, un proceso altamente dependiente de la generación de inferencias. Un componente clave de esta perspectiva es que las inferencias no solo posibilitan la realización práctica de conexiones entre las diferentes unidades semánticas del discurso escrito, sino que, además, son determinantes en el proceso de integración de los significados del discurso escrito en la estructura cognitiva del lector.

Se sabe que Bruner (1957), citado por León (2001), comparaba a la mente humana con una "máquina de inferencias" al referirse a su destreza para activar el conocimiento ya almacenado y utilizarlo para organizar e interpretar la nueva información entrante a través de complejas relaciones abstractas no provenientes de los estímulos. Esta idea sigue formando parte de la concepción actual sobre inferencias y el papel que juegan en la comprensión. Es así que hasta la actualidad se asume que cualquier proceso de comprensión del discurso conlleva un fuerte componente inferencial presente tanto en el dominio local del procesamiento de oraciones como en el dominio global del discurso.

Por eso, la comprensión lectora depende de que el lector infiera información. Bruning, Schraw y Ronning (2002) mencionan las investigaciones de Raphaely McKinney, 1983; Raphaely Pearson, 1982; y Raphael y Wonnacott, 1985. Los resultados indicaron que la capacidad de inferir de los estudiantes acerca del material del texto mejoraba su comprensión de modo significativo. Just y Carpenter (1992), Engle, Kane y Tuholsky (1999), citados por Barreyro y Molinari (2006) consideraron la participación de la memoria de trabajo y los recursos cognitivos en el procesamiento durante la lectura.

La trascendencia de la construcción de inferencias en la lectura y su relación con la comprensión lectora ha sido estudiada por diversos investigadores. Entre ellos destacan Trabasso (1977), Garrod y Sanford (1977), van Dijk y Kintsch (1983),
Just y Carpenter (1987), Graesser y Bower (1990), Kintsch (1990), van den Broek (1990). Cada uno de los autores mencionados y otros han contribuido con sus aportes a esclarecer diferentes aspectos de las inferencias como el objeto de la inferencia, la forma de inferir, la direccionalidad, las tipologías y la generación de inferencias y las tipologías. Es así que todas las investigaciones relacionadas con este tema han concluido en la importancia del nivel inferencial en la competencia lectora.

De acuerdo con León (2003), las inferencias son responsables de establecer coherencia local y global al texto. Las inferencias locales hacen referencia a las relaciones internas entre dos frases o palabras (género, número, tiempos verbales y complementos). Estas parecen realizarse durante la misma lectura; mientras que las globales se encargan de segmentos más amplios del texto como párrafos o ideas principales (quién, cómo, dónde, por qué) y se realizan con posterioridad a la culminación de la lectura y reorganización de la información en nuestra mente. Para el autor, no existen inferencias más importantes que otras. Cada texto promueve la realización de cierto tipo de inferencias, pero los textos narrativos permiten la mayor cantidad de estas.

Un texto está formado por proposiciones que se relaciona entre sí por medio de lazos formales explícitos que ayudan a determinar su significado. Cooper, citado por Maqueo (2004, p.73), sostiene la necesidad de que los estudiantes reconozcan dos distintas superestructuras: textos narrativos y expositivos. Frente a este aspecto, el arribo de las nuevas Tecnologías de la Información y la Comunicación (TIC) ha modificado las prácticas de lectura de manera significativa. De esta manera, se menciona al libro electrónico que es el nuevo medio de comunicación, después del texto impreso. Chartier, citado por Torres (2012), afirmó que leer en pantalla tiene característica principal el contar con destreza digital (búsqueda de información, manipulación de bases de datos, etc.).

Aunque los nativos digitales frecuenten la red y dominen su manejo, tienen limitaciones importantes para usar la red de manera significativa. Esto lo ha 
reiterado Nicholas, citado por Cassany (2012, p. 27), cuando dice que los adolescentes no siempre son conscientes de sus necesidades de información, por eso dependen de que se les ayude a investigar en fuentes fidedignas. Además, dedican poco tiempo a leer los textos digitales completos y a evaluatlos críticamente porque no son capaces de distinguir información confiable. También, desconocen los sistemas de almacenaje y recuperación de datos o el lenguaje que utilizan (términos clave, sintaxis de los motores de búsqueda, etc.). Incluso navegan por internet ignorando su estructura jerárquica o la calidad de los materiales. Por ejemplo, confunden una página web con un blog, una cuenta en una red social o un portal web.

Finalmente, se sabe por estudios de Nicholas (2007), citado por Cassany (2012), que los jóvenes navegan por internet siempre que pueden, suponen que toda la información necesatia está en la red, practican el plagio de información porque dejan de lado la publicación de las referencias utilizadas, y se sienten más cómodos con los formatos audiovisuales.

La relación de la inferencia con la lectura en textos impresos ha sido un tema recurrente incluso desde la aparición de los textos digitales. Por eso, Romo y Villalobos (2009) demostraron que hay menor comprensión durante el proceso de lectura si los estudiantes utilizan el texto digital en lugar del texto impreso. La causa de este resultado fue el uso de hipervínculos, propios de un texto en Internet, el cual disminuye la comprensión del texto, ya que el lector emplea mayor atención dividida y altenna, y disminuye la sostenida. Esta investigación es televante porque los programas educativos actuales utilizan diversos medios tecnológicos, y la habilidad lectora es importante para la construcción del conocimiento. Además, analizar las diferencias en la comprensión lectora entre el texto impreso y digital contribuye a entender mejor los procesos mentales implicados en la lectura y a establecer estrategias de mejora de los materiales didácticos para la competencia lectota.

En el Perú, el Acuerdo Nacional (2011-2016) y la Ley General de Educación 28044 proponen que el sistema educativo garantice que todos los estudiantes logren aprendizajes significativos y relevantes; sin embargo, es necesatio que estos estén definidos y ejemplificados de la maneta más clara posible, y que todos los ottos instrumentos de la política educativa se orienten a su logro. Es esta la razón que el Instituto Peruano de Evaluación, Acreditación y Certificación de la calidad de la Educación Básica (IPEBA) ha elaborado estándares de aprendizaje escolar, siguiendo las recomendaciones del Proyecto Educativo Nacional. Los estándares nacionales de aprendizaje ban sido elaborados como mapas de progreso, debido a que estos permiten describir claramente la secuencia en que progresan los aprendizajes fundamentales a lo latgo de la trayectoria escolar (Ministerio de Educación, IPEBA, 2013, Mapas de progreso, del aprendizaje. Nuestros estándares nacionales de aprendizaje, pp. 4-5).

De acuerdo con los estándares de aprendizaje del nivel $\mathrm{V}$, un estudiante egresado del $5^{\circ}$ grado de educación secundaria:

Lee comprensivamente textos con estructuras complejas que desarrollan temas diversos con vocabulario variado y especializado. Integra información contrapuesta o ambigua que está en distintas partes del texto. Interpreta el texto integtando la idea principal con información televante y de detalles. Evalúa la efectividad de los argumentos del texto y el uso de los tecursos textuales a partir de su conocimiento y del contexto sociocultural en el que fue escrito (Ministerio de Educación, IPEBA, 2012, p. 20).

En tal sentido, la aspitación de la educación peruana es que el estudiante, al finalizar la educación básica sea un lector competente porque realiza desempeños como los siguientes:

1. Explica ideas ambiguas, contradicciones y posturas poco claras del texto.

2. Compara con actitud crítica textos que presentan una variedad de posturas sobre el mismo tema.

3. Evalúa si el lenguaje empleado ayuda a lograt el objetivo que se propone el autor del texto.

4. Evalúa si la organización de la información es la más conveniente para lograr el objetivo que se propone el autor del texto. 


\section{UNIFÉ - EPG}

5. Explica si los argumentos expresados por el autor son suficientes para sustentar su idea.

6. Analiza la vigencia de las ideas del texto en el contexto sociocultural actual.

7. Explica el mensaje subliminal de un texto publicitario a partir de los recursos textuales e iconográficos.

A estos desempeños, se les puede agregar los que ya debieron lograr al terminar el ciclo anterior:

1. Explica las relaciones entre las actitudes de personajes de textos literarios y el sentido global de los mismos.

2. Argumenta su punto de vista frente al contenido del texto utilizando información que extrae del texto, de su conocimiento formal y de su experiencia.

3. Explica las características distintivas de los textos según su función y estructura (argumentativa, instructiva, etc.).

4. Propone alternativas de solución a problemas planteados en los textos, considerando el contexto sociocultural propio.

5. Explica el propósito comunicativo de las distintas partes del texto.

De lo expuesto, se puede afirmar que la presente es una investigación educacional con carácter pedagógico, centrada fundamentalmente, en la comprensión textual en el nivel inferencial de alumnos expuestos a la lectura de libros impresos y digitales. Este interés se investiga en diferentes contextos y en sujetos con distintos niveles intelectuales.

\section{Método}

Nivel de investigación

El nivel de investigación es descriptivo y de comprobación de las hipótesis causales, puesto que se recopiló información sobre el estado actual de un fenómeno con el fin de describirlo. El tipo de investigación es sustantiva. El objetivo fue examinar los efectos de la variación del formato de texto en la comprensión lectora. El diseño es pre-experimental, denominado "diseño de comparación estática o comparación de grupos con solo postest".

\section{Participantes}

La población estaba compuesta por 80 estudiantes del $4^{\circ}$ grado de secundaria de una institución educativa estatal mixta ubicada en la zona de Campoy del distrito de San Juan de Lurigancho, correspondiente a la UGEL 05 de Lima Metropolitana. La muestra estaba constituida por 38 estudiantes. Esta se determinó de manera intencional mediante el test de matrices progresivas de Raven con la finalidad de seleccionar a los estudiantes que presentan un nivel de inteligencia intermedio.

\section{Instrumentos}

1. Test de matrices progresivas de Raven (Inteligencia)

2. Prueba de Comprensión de textos en el nivel inferencial: elaborada por la investigadora. Mide la comprensión lectora en el nivel de las inferencias locales y globales.

3. Escala Motivacional de Lectura Académica (EML.A) de Ferreira, Muñoz, Sánchez, Santander, Pérez y Valenzuela (2012) y adaptado para estudiantes de educación secundaria para medir el hábito lector.

4. Instrumento de Evaluación de Competencias Digitales para Adolescentes en Riesgo Social: elaborado por Ballsells, Carrera y Vaquero (2011). Mide el nivel de la competencia digital.

\section{Técnicas de procesamiento y análisis de datos}

Las variables que se seleccionaron en esta investigación fueron la comprensión lectora en el nivel inferencial local y global como variable dependiente; el formato de texto (impreso $\mathrm{y}$ digital) como variable independiente; mientras que inteligencia, conocimiento previo, hábito lector y familiaridad con los textos digitales fueron las variables intervinientes. Se analizó la interacción de la comprensión lectora inferencial (global y local) con los formatos de texto impreso y digital. Asimismo se analizó la asociación de las variables de estudio con las variables intervinientes. 
Una vez que el grupo se hizo homogéneo en inteligencia se inició el experimento. Cada sección lee el texto en formatos diferentes y, después de una semana, se invierten los formatos en las secciones. Al finalizar la lectura, los estudiantes responden la evaluación inferencial en formato impreso y en digital. Pata este último se empleó la plataforma www.edmodo. com la cual fue creada en 2008 por Nic Borg y Jeff OHara. Es una herramienta que conecta estudiantes con profesotes. Esta ted social educativa, similar a la página de Facebook, permite a los estudiantes tener un aprendizaje participativo a través del desartollo de pruebas on line, enlaces a lecturas, almacenamiento de documentos, foros de discusión, etc. Los textos digitales se leyeron en formato PDF y, junto con las preguntas, se desartrollaton en las laptop XO 1.5 de color azul, que se utilizan en las escuelas públicas del nivel secundario.

Se hizo uso de la estadística descriptiva necesaria (media aritmética, mediana, desviación estándar y porcentaje de logto) de los indicadores de comprensión inferencial, hábito lector, competencia digital. Asimismo, se utilizó el gráfico de barras. Para la verificación de hipótesis se utilizó el Test "t" de Student para Medias Independientes.

\section{Resultados}

Comprensión inferencial y conocimiento previo

Esta investigación tiene como propósito determinar si el rivel de comprensión inferencial de textos es afectado por el nivel de conocimiento previo de los estudiantes. Para ello, se usó el promedio de las notas de los años anteriores de estudios. Dichas notas sirvieron para clasificat a los estudiantes en los niveles de conocimientos previos preestablecidos con anterioridad en la investigación. Los niveles de conocimientos previos a medir son conocimientos previos que se lograron y conocimientos previos en proceso de logro. Los resultados obtenidos se detallan a continuación. La prueba de Comprensión Lectora se aplicó en dos formatos: impreso y digital tomando en cuenta las inferencias locales y globales.

En la tabla 1 se analizan los resultados de las inferencias local y global, en los formatos impreso y digital, comparando los niveles alcanzados por los alumnos pertenecientes al grupo que logró los aptendizajes y al grupo que se encuentra en proceso según los resultados de los promedios de sus conocimientos previos. Para determinar si estas diferencias son significativas y utilizando también los valores del total, se aplicó el Test " $t$ " de Student para Medias Independientes.

Tabla 1

Resultados de la aplicación del Test "t" para medias anitméticas independientes entre los puntajes de inferencia, seguin formato, de sujetas de conocimiento previo diferente.

\begin{tabular}{|c|c|c|c|c|c|}
\hline Formato & Inferencia & tc & $\begin{array}{c}\mathrm{t}_{5} \\
05,36\end{array}$ & Critetio & Decisión \\
\hline \multirow{2}{*}{ Impreso } & Local & 0.089 & \multirow{4}{*}{2.031} & \multirow{4}{*}{$\begin{array}{c}\text { "Si tc } \geq t, \\
, g d \\
\Rightarrow>\text { Rechazar } \\
H_{0} "\end{array}$} & $\begin{array}{c}\text { Falla } \\
\text { rechazar } \\
\mathrm{H}_{\mathrm{a}}\end{array}$ \\
\hline & Global & 0.624 & & & $\begin{array}{c}\text { Falla en } \\
\text { techazar } \\
\mathrm{H}_{n}\end{array}$ \\
\hline \multirow[t]{2}{*}{ Digigal } & Local & 3.089 & & & $\begin{array}{l}\text { Falla err } \\
\text { rechazar } \\
\mathrm{H}\end{array}$ \\
\hline & Global & 1.515 & & & $\begin{array}{l}\text { Falla } \\
\text { en } \\
\text { trechazar } \\
\mathrm{H}_{0}\end{array}$ \\
\hline
\end{tabular}

Como se puede observar en la tabla 1, en tres comparaciones de los puntajes de los dos grupos de niveles de conocimiento previo, el valor del " $\mathrm{t}$ " empítico (tc) del Test " $t$ " no es mayor ni igual que el valor del " $\mathrm{t}$ " teótico por lo que se falla en rechazar la hipótesis nula, Ho. Esto significa que no existe diferencia significativa entre los puntajes obtenidos por los niveles de conocimiento previo en las inferencias locales y globales del fotmato impreso y en la inferencia global del formato digital. Por lo tanto, se puede afirmar que ambos grupos presentaban un mismo nivel de inferencia local y global.

Por otro lado, en una comparación, el valor del " $\mathrm{t}$ " empírico (tc) del Test " $\mathrm{t}$ " de Student es mayor que el valor del "t" teórico, por lo que se rechaza la hipótesis nula, Ho. Esto significa que sí existe diferencia significativa entre los puntajes obtenidos por los niveles de conocimiento previo de una inferencia local. 


\section{UNIFE-EPG}

Por lo tanto, se puede afirmar, que los puntajes de la inferencia local, del formato digital, del grupo de mayor conocimiento previo superan significativamente al de menor conocimiento previo.

Con relación a la subhipótesis 1.1 que establecía que el nivel de comprensión inferencial de textos es afectado por el nivel de conocimiento previo de los estudiantes, no ha sido confirmada por cuanto de cuatto situaciones, en tres de ellas, se falla en rechazar.

Comprensión inferencial y hábito lector

Como se puede observar en la Tabla 2, el valor del "t" empírico (tc) del Test " $\mathrm{t}$ " no es mayor ni igual que el valor del " $\mathrm{t}$ " teórico por lo que se falla en rechazar la hipótesis nula, Ho en todos los casos. Esto significa que no existe diferencia significativa entre los puntajes obtenidos por los dos grupos según el hábito lector, en las inferencias locales y globales del formato impreso y del digital. Por lo tanto, se puede afirmar que ambos grupos de hábito lector presentaban un mismo nivel de inferencia local y global en los dos formatos.

Con relación a la subhipótesis 1.2 que establecía que el hábito lector de los estudiantes afecta positivamente el nivel de comprensión inferencial de textos, esta no ha sido confirmada en ningún caso.

Tabla 2

Resultados de la aphicación del Test "i" para medias anitméticas independientes entre los puntajes de inferencia, seguin formato de sugietos de bábito lector diferente.

\begin{tabular}{|c|c|c|c|c|c|}
\hline Formato & Inferencia & tc & $\begin{array}{c}t_{3} \\
05,36\end{array}$ & Cistetio & Decisión \\
\hline \multirow[b]{2}{*}{ Impreso } & Local & 1.3067 & & & $\begin{array}{c}\text { Fallat en } \\
\text { rechazar } \\
\mathrm{H}_{*}\end{array}$ \\
\hline & Global & 0.2471 & & $\begin{array}{c}\text { "Si tc } \geq \varepsilon, \\
\text {,gl. }\end{array}$ & $\begin{array}{c}\text { Fallat en } \\
\text { rechazar } \\
\mathrm{H}_{0}\end{array}$ \\
\hline \multirow[b]{2}{*}{ Digital } & Iocal & 0.2421 & 2.031 & $\begin{array}{c}\Rightarrow \text { Rechazar } \\
\mathrm{H}_{13}^{\prime \prime}\end{array}$ & $\begin{array}{l}\text { Fallat en } \\
\text { rechazar }\end{array}$ \\
\hline & Global & 0.0783 & & & $\begin{array}{c}\mathrm{H}_{0} \\
\text { Fallar en } \\
\text { techazat } \\
\mathrm{H}_{0}\end{array}$ \\
\hline
\end{tabular}

Comprensión inferencial y competencia digital
Como se puede observar en la tabla 3, en tres comparaciones, el valor del "t" empírico (tc) del Test " $\mathrm{t}$ " no es mayor ni igual que el valor del "t" teórico por lo que se falla en rechazar la hipótesis nula (Ho). Esto significa que no existe diferencia significativa entre los puntajes obtenidos por los dos grupos según competencia digital, en las inferencias locales y gobales del formato digital, y en la inferencia local del formato impreso. Por lo tanto, se puede afirmat que ambos grupos de competencia digital presentaban un mismo nivel de inferencia local y global.

Por otro lado, en una comparación, el valor del " $e$ " empírico (tc) del 'Test " $t$ " de Student es mayor que el valor del "" teótico, por lo que se rechaza la hipótesis nula, Ho. Esto significa que sí existe diferencia significativa entre los puntajes obtenidos por los dos grupos de competencia digital de una infetencia global. Por lo tanto, se puede afirmar, que los puntajes de la inferencia global, del formato impreso, del grupo de Perfil Alto superan significativamente al del Perfil Medio.

Con relación a la subhipótesis 1.3 que establecía que la competencia digital afecta diferencialmente el nivel de comprensión inferencial cuando se usa el formato digital, esta no ha sido confirmada.

\section{Tabla 3}

Resultados de la aplicación del Test "I" para medias aritméticas independientes entre los puntajes de inferencia, seguin formato de sujetos de competencia digital diferente.

\begin{tabular}{|c|c|c|c|c|c|}
\hline Formato & Inferencia & tc & $\begin{array}{c}\mathrm{t}, \\
05,36\end{array}$ & Critenio & Decisión \\
\hline \multirow[b]{2}{*}{ Impreso } & Local & 1.1410 & & \multirow{4}{*}{$\begin{array}{c}\text { "Si } t c \geq t, \\
\text { gl. } \\
\Rightarrow \text { Recthazar } \\
\mathrm{H}_{0} \text { " }\end{array}$} & $\begin{array}{c}\text { Iaallar en } \\
\text { rechazat } \\
\mathrm{H}_{0}\end{array}$ \\
\hline & Global & 2.4387 & & & $\begin{array}{c}\text { Fallar en } \\
\text { rechazar } \\
\mathrm{H}\end{array}$ \\
\hline \multirow[b]{2}{*}{ Digitall } & I.ocal & 0.9211 & 2.031 & & $\begin{array}{l}\text { Fallat en } \\
\text { rechazarar }\end{array}$ \\
\hline & Global & 1.5478 & & & $\begin{array}{c}\mathrm{H}_{0} \\
\text { Fallat en } \\
\text { rechazar } \\
\mathrm{H}\end{array}$ \\
\hline
\end{tabular}

Niveles de inferencia y soporte de presentación del texto según formato 
Como se puede observar en la tabla 4, el valor del " $\mathrm{t}$ " empirico (tc) del Test " $\mathrm{t}$ " no es mayor ni igual que el valor del " $\mathrm{t}$ " teórico por lo que se falla en rechazar la hipótesis nula, Ho. Esto significa que no existe diferencia significativa entre los puntajes obtenidos en las inferencias locales y globales entre los formatos impreso y digital, así como en el nivel inferencial en ambos tipos de formato: impreso y digital. Con relación a la subhipótesis 1.4 que establecía que los niveles de inferencia local, inferencia global y nivel inferencial varian según el soporte de presentación del texto, esta no ha sido confirmada.

\section{Tabla 4}

Resultados de la aplicación del Test "t" para medias animéticas independientes entre los puntajes de inferencia, segin tipo de formato.

\begin{tabular}{|c|c|c|c|c|}
\hline I Tormato & tc & $\begin{array}{c}t_{7} \\
05,74\end{array}$ & Cuterio & Decisión \\
\hline I & 0.2415 & & & Fallar en rechazar \\
\hline Gilobal & 0.4874 & 1.99 & $\begin{array}{c}{ }^{c} \mathrm{Si} t \mathrm{c} \geq \mathrm{t}, \\
\mathrm{gl} \\
\Rightarrow \text { Rechazar }\end{array}$ & $\begin{array}{c}\mathrm{H}_{\mathrm{t}} \\
\text { Erallar en rechazat } \\
\mathrm{H}_{0}\end{array}$ \\
\hline Inferencial & 0.0034 & & & $\begin{array}{c}\text { Fallat en techazat } \\
\mathrm{H}_{\mathrm{r}}\end{array}$ \\
\hline
\end{tabular}

\section{Discusión}

En cuanto al supuesto que establece que el nivel de comprensión inferencial de textos es afectado por el nivel de conocimiento previo de los estudiantes, puede decirse que no ha sido confirmado porque se rechpza la Ho en tres situaciones de las cuatro que se han presentado.

Para una evaluación más equitativa, se ha considerado Ios promedios escolares de los tres años anteriores como saberes previos de ambos grupos. Así se tuvo que el grupo que había logrado su aprendizaje tenía calificaciones entre 14 y 16 y el grupo que estaba en proceso entre 11 y 13 . Se ha observado que los resultados obtenidos pudieton haber sido diferentes si los puntajes en los conocimientos previos hubieran sido tnás extremos entre ellos; por ejemplo, si en lugar de calificaciones del grüpo que logró el aprendizaje hubiera sido entre 19 y 20 , y no, 14 y 16.
En primer lugar, tanto el grupo que se hallaba en proceso como el grupo que ya había logrado sus aprendizajes, presentaron un mismo nivel de inferencia local y global. Esto se debió a que los temas de las lecturas que se aplicaron en la evaluación son de su contexto. Así se tiene que la primera lectura titulada El sueño del pongo es del escritor peruano José Maria Arguedas, de quien ya habia leído otras obtas como Yawar Fiesta o El sexto. Además, en el colegio se viene promoviendo la sensibilización e interés por el medio ambiente, tema de la segunda lectura Las abejas desaparecen. por qué debemos preocuparnos.

En segundo lugat, los saberes previos incluyen conocimientos específicos; pero también habilidades, actitudes, formas de comunicar, escuchar, aprender y enseñar. Son pues, saberes que permiten ser/estar en el mundo y suponen diversos niveles de complejidad. Entonces, los saberes previos no solo se pueden remitir a los promedios de las evaluaciones de los años anteriores, sobre todo, si en la institución educativa se sigue enseñando en base a la memorización de contenidos y no en base al desarrollo de competencias. Prueba de esta observación es que el máximo puntaje del promedio de los tres años es de 16 en la escala vigesimal.

En tercer lugar, de acuerdo con Alliende y Condemarín (p. 34), el hogar y la comunidad determinan el nivel de estimulación lingüística, así como las actitudes frente a la lectura, los modelos de padres lectores y la lectura por obligación o como castigo, inculcados en el hogar. Los estudiantes evaluados leen en su mayoría diarios informales, cuya vatiación lingǘstica es subestándar, debido a su bajo costo y a temas relacionados con la farándula y criminalística.

Pot otro lado, se ha coincidido con los resultados de la investigación de Rosalvina Perez (2010, p. 17) quien luego de aplicar un método interactivo para mejorar la lectura y de tomar en cuenta los saberes previos en un grupo experimental, concluyó que la diferencia entre este grupo y el de control no fue muy significativa.

En cuanto al supuesto que establece que el hábito lector de los estudiantes afecta positivamente el 


\section{UNIFÉ - EPG}

nivel de comprensión inferencial de textos no ha sido confirmado. Esto también se debe a que puntajes no son muy extremos entre sí.

La explicación estadística a este resultado se fundamenta en que se debió aplicar varias pruebas para una mejor observación de los datos. Además, el número de sujetos con los que se contó fue pequeño. Esto es consecuencia de la clasificación que se hizo según los resultados de la prueba de Raven.

Por otro lado, se ha concluido en que los grupos de nivel alto y el de nivel medio de hábito lector presentan un mismo nivel de inferencia local y global en los dos tipos de formato impreso y digital. Esto demuestra que los estudiantes comprenden los textos por ser de su contexto y porque no son muy extensos; además, el tiempo con el que tuvieron para desarrollar la prueba que fue de 90 minutos. Otra razón de este resultado coincide con la cuarta conclusión de Albarello (2011, p. 216), la cual aparece en el marco teórico referente al numeral 4.15, el cual menciona que la interactividad lleva a la distracción y la poca concentración de la lectura; y en la prueba aplicada en formato digital, solo podían acceder al texto para luego responder las preguntas de manera virtual. En el mismo numeral, puede verificarse en la séptima y octava conclusión de Albarello (2001, p. 217) las cuales se refieren a que se pueden realizar estrategias de lectura no necesariamente de texto no lineales, y que las estrategias de lecturas son establecidas de acuerdo con el interés del lector, en este caso, interés académico.

Con relación al supuesto que establece que la competencia digital afecta diferencialmente el nivel de comprensión inferencial cuando se usa el formato digital, puede decirse que este ha sido confirmado parcialmente solo en un caso de cuatro.

El resultado de que los estudiantes tienen un nivel de comprensión lectora aceptable en ambos tipos de inferencia tanto local como global y en los dos formatos: impreso y digital, se apoya en la afirmación de Cassany (2012, p. 25) quien cuestiona las ideas de Mark Prensky sobre los nativos digitales. Cassany ha observado que en la vida cotidiana, ambos formatos (impreso y digital) se complementan, así se puede apreciar cuando se lee diarios, mensajes de texto, facturas de cuentas, letreros luminosos en las calles, señales de tráfico, publicidad televisiva o impresa, etc. incluso, los estudiantes llegan al colegio con una experiencia digital variada. En ese caso, insiste el autor, no debe confundirse el acceso a la red con el uso adecuado del internet para prácticas académicas. Nicholas et al. (2007), citado por Cassany (2012, p. 27) afirma que los adolescentes no siempre dedican suficiente tiempo a leer textos digitales completos y a evaluarlos críticamente, incluso no son capaces de reconocer información confiable. También desconoce los sistemas de almacenaje y recuperación de datos, y navegan por internet de manera ingenua, ignorando la estructura jerárquica de los textos en pantalla.

Esta última aseveración coincide con los resultados de la aplicación del Instrumento de Evaluación de Competencias Digitales para Adolescentes en Riesgo Social de Balsells, Carrera y Vaquero (2011), donde el resultado más alto se apreció en las preguntas del uso de las redes sociales, mientras que los resultados más bajos se obtuvieron en las preguntas concernientes a la ofimática y a las actitudes frente al uso correcto del Internet. Sin embargo, los sujetos de evaluación pertenecen a una generación que ha incorporado el uso de los textos digitales en su vida cotidiana. Es por eso que Torres (2012), como se indica en el marco teórico, sostiene que una lectura digital exige habilidad para manipular dispositivos electrónicos, la cual puede también ser aprendida por los "migrantes digitales", aunque no con la misma facilidad que los "nativos digitales".

Con relación al supuesto que establece que los niveles de inferencia local, inferencia global y nivel inferencial varían según el soporte de presentación del texto, no ha sido confirmado. Lo mismo sucede con la hipótesis general.

Hay una diferencia mínima en los resultados de la Prueba de Comprensión Lectora en la inferencia local y en la inferencia global. También cabe precisar que los puntajes obtenidos en los dos tipos de formato no son muy distantes entre sí.

Por otro lado, se puede afirmar que en la inferencia global, los resultados en ambos tipos de 
Alétheia 2015,3 (1) 69

formatos (impreso y digital) son mayores que en la inferencia local.

Inferit es extraer información implícita de un texto a partir de datos explícitos. La inferencia gobal es aquella que se realiza a partir de la lectura total de un texto, mientras que la inferencia local se concentra solo en una parte del texto. De alli que inferir de manera giobal es una habilidad altamente compleja, ya que necesita abstraer las ideas principales de cada párrafo y del texto global. Además, previamente se debe reconocer la tipología textual y sus procedimientos discursivos. Estas habilidades han sido demostradas por la mayoría de los estudiantes al responder casi la totalidad de las preguntas de la prueba de comprensión lectora de manera correcta.

Por lo tanto, la muestra sometida a evaluación ha demostrado tener esa habilidad, resultado que coincide con las hipótesis anteriores por cuanto ni los saberes previos ni el hábito lector ni la competencia digital han inftuido en la comprensión lectora.

\section{Conclusiones}

La ptesente investigación permite elaborar las conclusiones que se expresan a continuación.

1. Los saberes previos son importantes en la comprensión de lectura si se considera como situaciones de espacio o tiempo, condiciones de los petsonajes o significados de expresiones o frases que son parte del contexto de la lectura y del mundo del autor; pero no son determinantes para una comprensión del texto cuando los estudiantes pueden inferir correctamente si toma en cuenta los datos explícitos del mismo.

2. La prueba para medir el hábito lector fue una adaptación de un instrumento para medir la motivación por la lectura académica. Se tomó esta decisión, debido a que la mayoría de los alumnos leen textos, generalmente, con temáticas relacionadas a los cursos que estudian. Aunque los resultados de esta prueba permitieton separar a la muestra en dos grupos: alto y medio, según el interés por leer, los puntajes fueron bajos. Es por eso que se concluye que las lecturas, para una mejor comprensión de las mismas, deben ser parte de la realidad de los estudiantes. De esta manera, se logxa que ellos se interesen por leer de maneta intrínseca. El hábito lector se forma desde el hogat y no debe imponerse en los colegios como parte de una nota.

3. La competencia digital no afecta de manera significativa el nivel de comprensión inferencial de los estudiantes. Esta conclusión se debe a que una mayoría desconoce y no utiliza una computadota y los recursos de la web 2.0 para mejorar su aprendizaje. Por el contrario, prefieren el empleo de las redes sociales y dispositivos como celulares, laptop, tablets o pendrive (USB) para actividades lúdicas como videojuegos, almacenamiento de música o comunicaciones coloquiales con los amigos.

4. Esta investigación concluye en que el nivel inferencial, tanto global como local, permite una mejor comprensión del texto leído; y que no ha inferido en su medición los formatos empleados o el interés por lecturas académicas o dominio de competencias digitales.

\section{Referencias}

Albarello, F. (2011). Leer/ Navegar en Internet. Las formas de lectura en la computadora. Buenos Aires, Argentina: La Crujía.

Alliende, F, y Condemarín, M. (1990). La lectura: Teoría, evaluación y desarrollo. Santiago, Chile: Editorial Andrés Bello.

Artopoulos, A. (2010). De la computadora "florero" al celub-bot: sobre la difusión de tecnologías en educación. En: Escuela y TICs: los caminos de la innovación. Buenos Aires, Argentina: Lugar editorial.

Ausubel, D. (2002). Adquisición y retención del conocimiento: una perspectiva cognitiva (G. Sánchez, trad.). Barcelona, España: Paidós.

Balsells, M., Carrera, F., y Vaquero, E. (2011). Instrumento de evaluación de competencias digitales para adolescentes en riesgo social. 
70

Edutec-e. Revista electrónica de Tecnología Educativa, 35, 1-25.

Barreyro,J.yMolinari,C.(2006).Diferenciasindividuales en la comprensión de textos: inferencias y capacidad de la memoria de trabajo. Buenos Aires, Argentina: Universidad Autónoma de Buenos Aires. Recuperado de: http:// www.scielo.org.ar/scielo.php?pid=S1851 $16862006000100034 \&$ script $=$ sci_arttext

Bruning, R., Schraw, G., y Ronning, R., (2002). Psicología cognitiva e instrucción (C. Gonzales, trad.). Madrid, España: Alianza Editorial.

Camargo, J. (2008). El libro electrónico. La industria editorial en la era de la revolución digital. Bogotá: Pontificia Universidad Javeriana. Recuperado de: http://www.javeriana.edu.co/biblos/tesis/ comunicacion/tesis19.pdf

Cassany, D. (2012). En_línea. Leer y escribir en la red. Barcelona, España: Anagrama.

Cassany, D. (Enero 2013). ¿Sabemos leer en la red? Revista cientifica Investigación y ciencia. $N^{\circ}$ 436. Recuperado de: http://www. investigacionyciencia.es/files/11967.pdf

Cassany, D. (Abril, 2013). ¿Cómo se lee y escribe en línea? Revista electrónica Leer, escribir y descubrir. (1)1, 1-24. Recuperado de: http://www.reading.org/Libraries / international/revista-electronica-leerescribir-y-descubrir2.pdf

Cordón, J. (2011). I a revolución del kibro electrónico. Barcelona, España: UOC.

Escoriza, J. (2003). Evaluación del conocimiento de estrategias de comprensión lectora. Barcelona, España: Universidad de Barcelona.

Ferreira, S., Muñoz, C., Sánchez, P., Santander, S., Pérez, M., Valenzuela, J., (2012). Características psicométricas de una escala para caracterizar la motivación por la lectura académica REDIE. Revista Electrónica de Investigación Educativa, 14 (2), 118-132.

Galina, I. (2002). I a lectura en la era digital. México, D.F., México: Universidad Nacional Autónoma de México. Recuperado de: http://www.redalyc. org/pdf/285/28550103.pdf
Gárate, M., Gutiérrez, F., Luque, J., García, J., y Elosúa, M. (1999). Inferencias y comprensión lectora. En: García, J., Elosúa, M., Gutierrez, F., Luque, J., y Gárate, M. Comprensión lectora y memoria operativa. Barcelona, España: Paidós, p. 42.

García, E., y García, L. (2001). I a biblioteca digital. Madrid, España: Arco libros.

Gobierno de España, Biblioteca Nacional de España. (2010). Del códice al libro electrónico. Madrid, España: Autor. Recuperado de: http:// www.bne.es/es/Actividades/Exposiciones/ Exposiciones/docs/Folleto_Del_codice_al_ libro_digital.pdf

Gonzales, J. (1991). Las inferencias durante el proceso lector. En Puente, A. (dir.). Comprensión de la lectura y acción docente. Madrid, España: Fundación Germán Sanchez Rupérez, p. 111, 124.

Grossman, L. (07 de noviembre de 2011). Del rollo a la pantalla. Madrid, España: El Cultural Electrónico, S.L. Recuperado de: http://www. elcultural.es/version_papel/LETRAS/29854/ Del_rollo_a_la_pantalla

Inga, M. (Julio - Diciembre 2010). Investigación del papel de la memoria operativa, la inferencia y la competencia gramatical en la compresión lectora. Investigación educativa, 14(26), 51-66.

Kispal, A. (2008). Effective teaching of inference skills for reading. Literature Review. National Foundation for Educational Research. ISBN 9781847751416 . Recuperado de: http://dera. ioe.ac.uk/7918/1/DCSF-RR031.pdf

León, J. A. (2001). Las inferencias en la comprensión e interpretación del discurso. Signos, 34(4950) ,113-115. Recuperado de: http://www. scielo.cl/scielo.php?script=sci_arttext\&pid =S0718-09342001004900008

León, J. A. (2003). Conocimiento y discurso. Claves para inferir y comprender. Madrid, España: Pirámide.

Maqueo, A. (2004). Lengua, aprendizaje y enseñanza. México, D.F., México: Limusa.

Millán, J. (2013). Leyendo pantallas. La metamorfosis de un artículo desde su publicación en un suplemento literario hasta su compresión en un 
tuit, pasando por webs, tabletas y teléfonos. En: Libros \& bitios. [Blog]. Recuperado de: http:// jamillan.com/librosybitios/leyenpan.htm

Ministerio de Educación, Instituto de Evaluación. (2010). La lectura en PISA 2009. Marros y pruebas de la evaluación. Madrid, España: Autor. Recuperado de: https://www.mecd.gob.es/ $\mathrm{dctm} /$ ievaluacion/internacional/lectura-enpisa.pdf?documentld=0901e72b8072f8d9

Ministerio de Educación, Instituto Peruano de Evaluación, Acreditación y Certificación de la calidad de la Educación Básica - IPEBA. (2013). Mapa de progreso del aprendizaje. Lectura. Lima, Penú: Autor. Recuperado de: ht»://wwwipeba.gob.pe/estandares/ MapasProgreso_Comunicacion_Lectura.pdf

Ministerio de Educación, Instituto Peruano de Evaluación, Acreditación y Certificación de la calídad de la Educación Básica - IPEBA. (2013). Mapas de progreso del aprendizaje. Nuestros estándares nacionales de aptendizaje. Lima, Perú: Autor. Recuperado de: http://ipeba.gob. pe/estandares/MapasProgresoPeru.pdf

Ministerio de Educación, Unidad de Medición de la Calidad. (Diciembre 2013). PISA 2012: Primeros resultados. Informe Nacional del Perí. Lima, Perú: Autor. Recuperado de: http://www2.minedu.gob.pe/umc/PISA/ Pisa2012/Informes_de_resultados/Informe PISA_2012_Peru.pdf

Monteoliva, E., Pérez-Ortiz, C., y Repiso, R. (2008). Lectores de documentos electrónicos. Granada, España: Escuela Superior de Comunicación. Recuperado de: http://cdcescogtanada.com/ cdc/wp-content/uploads/2009/03/2cuadern os108-119.pdf

Pinzás, J. (1995). Leet pensando. Introducción a la visión contemporánea de la lectura. Lima, Petú: Asociación de Investigación aplicada y Extensión Pedagógica Sofia Pinzás.

Pinzás, J. (29 de matzo de 2012). Las siete estrategias cognitivas del buen lector. [Diapositivas]. En Conferencia magistral a los Directores de Instituciones Educativas "Marca Perú".
Alétheia 2015,3 (1) 71

Dirección Regional de Lima (DREIM), Perú. Recuperado de: http://wwweducacionenred. com/Noticia/?portada=17991 y http:// wwe.educacionenred.com/?i=http://goo.gl/ NYfLM

Romo, A, y Villalobos, M. (2009). Comprensión lectora en texto impreso y digital: sus diferencias. Trabajo presentado en X Congreso Nacional de Investigación Educativa. México D.F., México. Recuperado de: http://www:comle.otg.mx/ congreso $/$ memoriaelectronica $/ \mathrm{v} 10 / \mathrm{pdf} / \mathrm{area}_{\text {- }}$ tematica_07/ponencias/0905-F.pdf

Romo, A., y Villalobos, M. (Enero, 2011). Hacia una nueva cultura: La comprensión en la lectura digital Revista científica Internacional Indexada ISSN 1679-9844. 16, 112-130.

Torres, M. (2012). Lectura en pantalla y las nuevas competencias de la era digital. Neuquén, Argentina: Universidad Nacional de Comahue. Recuperado de: http://seadiuncoma.files. wordpress.com/2012/06/017-la-lectura-enpantalla-y-las-nuevas-competencias-de-la-eradigital.pdf

Tortes, P. (2005). Tipos de inferencia en la comprensión lectora de alumnos de sexto grado. (Tesis de Maestría). Durango - México: Universidad Autónoma de Dutango. Recuperada de: http://enlacescardenal.bligoo.cl/media/ users $/ 17 / 859943 /$ files $/ 166073 /$ TIPOS_ INFERENCIAS_COM-LEC_2005.pdf 